\title{
Existence of Periodic Solutions to Nonlinear Differential Equations of Third Order with Multiple Deviating Arguments
}

\author{
Cemil Tunç \\ Department of Mathematics, Faculty of Sciences, Yüzüncü Yıl University, 65080 Van, Turkey \\ Correspondence should be addressed to Cemil Tunç, cemtunc@yahoo.com
}

Received 12 May 2012; Accepted 19 July 2012

Academic Editor: D. D. Ganji

Copyright (C) 2012 Cemil Tunç. This is an open access article distributed under the Creative Commons Attribution License, which permits unrestricted use, distribution, and reproduction in any medium, provided the original work is properly cited.

We establish certain new sufficient conditions which guarantee the existence of periodic solutions for a nonlinear differential equation of the third order with multiple deviating arguments. Using the Lyapunov functional approach, we prove a specific theorem and provide an example to illustrate the theoretical analysis in this work and the effectiveness of the method utilized here.

\section{Introduction}

It is known that functional differential equations, in particular, that delay differential equations can be used as models to describe many physical, biological systems, and so forth. In reality, many actual systems have the property aftereffect, that is, the future states depend not only on the present, but also on the past history, and after effect is also known to occur in mechanics, control theory, physics, chemistry, biology, medicine, economics, atomic energy, information theory, and so forth (Burton [1], Kolmanovskii and Myshkis [2]). Therefore, it is important to investigate the qualitative behaviors of functional differential equations.

In 1978, using the known theorem of Yoshizawa [3, Theorem 37.2], Chukwu [4] found certain sufficient conditions that guarantee the existence of a periodic solution to nonlinearlinear differential of the third order with the constant deviating argument $h(>0)$ :

$$
\begin{aligned}
x^{\prime \prime \prime}+ & f\left(x, x^{\prime}, x^{\prime \prime}\right) x^{\prime \prime}+g\left(x(t-h), x^{\prime}(t-h)\right)+i(x(t-h)) \\
& =p\left(t, x, x^{\prime}, x(t-h), x^{\prime}(t-h), x^{\prime \prime}\right) .
\end{aligned}
$$


Later, in 1992, Zhu [5] considered the nonlinear differential equation of the third order with the constant deviating argument $r(>0)$ :

$$
x^{\prime \prime \prime}+a x^{\prime \prime}+\phi\left(x^{\prime}(t-r)\right)+f(x)=p(t)
$$

and he discussed the existence of periodic solutions for this equation when $p(t)$ is a periodic function of period $T, T>0$.

In 2000, Tejumola and Tchegnani [6] considered the nonlinear differential equation of the third order with the constant deviating argument $\tau(>0)$ :

$$
\begin{aligned}
x^{\prime \prime \prime}+ & f\left(t, x, x^{\prime}, x^{\prime \prime}\right)+g\left(t, x(t-\tau), x^{\prime}(t-\tau)\right)+h(x(t-\tau)) \\
& =P_{1}\left(t, x, x^{\prime}, x^{\prime \prime}, x(t-\tau), x^{\prime}(t-\tau)\right) .
\end{aligned}
$$

The authors established certain sufficient conditions on the existence of periodic of solutions of this equation.

In 2010, Tunç [7] established certain sufficient conditions for the existence of a periodic solution for the nonlinear differential equation of the third order with the constant deviating argument $r(>0)$ :

$$
x^{\prime \prime \prime}+\psi\left(x^{\prime}\right) x^{\prime \prime}+g\left(x^{\prime}(t-r)\right)+f(x)=p\left(t, x, x(t-r), x^{\prime}, x^{\prime}(t-r), x^{\prime \prime}\right) .
$$

However, a review to date of the literature indicates that the existence of periodic solutions to the nonlinear differential equation of the third order with multiple deviating arguments has not been investigated. The paper considers the nonlinear differential equation of the third order with multiple constant deviating arguments $\tau_{i},(i=1,2, \ldots, n)$ :

$$
\begin{aligned}
x^{\prime \prime \prime}+ & \psi\left(x^{\prime}\right) x^{\prime \prime}+\sum_{i=1}^{n} g_{i}\left(x^{\prime}\left(t-\tau_{i}\right)\right)+f(x) \\
& =p\left(t, x, x\left(t-\tau_{1}\right), \ldots, x^{\prime}, \ldots, x^{\prime}\left(t-\tau_{n}\right), x^{\prime \prime}\right) .
\end{aligned}
$$

The equation (1.5) is stated in system form as follows:

$$
\begin{gathered}
x^{\prime}=y, \quad y^{\prime}=z \\
z^{\prime}=-\psi(y) z-\sum_{i=1}^{n} g_{i}(y)-f(x)+\sum_{i=1}^{n} \int_{t-\tau_{i}}^{t} g_{i}^{\prime}(y(s)) z(s) d s \\
+p\left(t, x, x\left(t-\tau_{1}\right), \ldots, y\left(t-\tau_{n}\right), z\right)
\end{gathered}
$$

where $\tau_{i}$ are positive constants, that is, $\tau_{i}$ are constant deviating arguments, which are determined in Section 2. It is assumed that the functions $\psi, g_{i}, f$, and $p(\cdot)$ are continuous in their respective arguments on $\mathfrak{R}, \mathfrak{R}, \mathfrak{R}$, and $\mathfrak{R}^{+} \times \mathfrak{R}^{2 n+3},\left(\mathfrak{R}^{+}=[0, \infty), \mathfrak{R}=(-\infty, \infty)\right)$, respectively; $g_{i}(0)=f(0)=0$ and $p(\cdot)$ is periodic in $t$ of period $T, T \geq \tau_{i}$, the derivatives 
$g_{i}^{\prime}(y) \equiv(d / d y) g_{i}(y)$ exist and are also continuous; throughout what follows $x(t), y(t)$, and $z(t)$ are abbreviated as $x, y$, and $z$, respectively.

The motivation for this paper is a result of the research mentioned regarding ordinary differential equations with a deviating argument. Our aim is to achieve the results established in $[5,7]$ to $(1.5)$ with multiple deviating arguments. Our results generalize the results established on the existence of periodic solution in $[5,7]$. This paper is the first known publication regarding the existence of periodic solution for differential equations of the third order with multiple deviating arguments.

In order to reach our main result, this paper offers fundamental information regarding the general nonautonomous delay periodic differential system. Consider the delay periodic system:

$$
\dot{x}(t)=F\left(t, x_{t}\right), \quad x_{t}=x(t+\theta), \quad-r \leq \theta \leq 0, \quad t \geq 0,
$$

where $F:[0, \infty) \times C_{H} \rightarrow \mathfrak{R}^{n}$ is a continuous mapping, $F(t+T, \varphi)=F(t, \varphi)$ for all $\varphi \in C$ and for some constant $T>0$. We assume that $F$ takes closed bounded sets into bounded sets of $\mathfrak{R}^{n}$. Here $(C,\|\cdot\|)$ is the Banach space of continuous function $\phi:[-r, 0] \rightarrow \mathfrak{R}^{n}$ with supremum norm, $r>0$; for $H>0$, we define $C_{H} \subset C$ by $C_{H}=\{\phi \in C:\|\phi\|<H\}, C_{H}$ is the open $H$-ball in $C, C=C\left([-r, 0], \Re^{n}\right)$.

Theorem 1.1. Suppose that $F(t, \varphi) \in \bar{C}_{0}(\varphi)$ and $F(t, \varphi)$ is periodic in $t$ of period $T, T \geq r$, and consequently for any $\alpha>0$ there exists an $L(\alpha)>0$ such that $\varphi \in C_{\alpha}$ implies $|F(t, \varphi)| \leq L(\alpha)$. Suppose that a continuous Lyapunov functional $V(t, \varphi)$ exists, defined on $t \in \mathfrak{R}^{+}, \varphi \in S^{*}, S^{*}$ is the set of $\varphi \in C$ such that with $|\varphi(0)| \geq H$ (H may be large), and that $V(t, \varphi)$ satisfies the following conditions.

(i) Continuous increasing functions $a(s)$ and $b(s)$ exist, satisfying $a(s)>0, b(s)>0$ for $s \geq H$ and $a(s) \rightarrow \infty$ as $s \rightarrow \infty$, such that

$$
a(|\phi(0)|) \leq V(t, \phi) \leq b(\|\phi\|) \text {, when }|\varphi(0)| \geq H .
$$

(ii) A continuous and positive function w(s) exists such that

$$
\dot{V}(t, \phi) \leq-w(|\varphi(0)|) \quad \text { for } s \geq H
$$

(iii) A constant $H_{1}>0, H_{1}>H$, exists such that

$$
r L\left(\gamma^{*}\right)<H_{1}-H,
$$

where $\gamma^{*}>0$ is a constant which is determined in the following way.

Using the condition on $V(t, \varphi)$, constants $\alpha>0, \beta>0$, and $\gamma>0$ exist such that $b\left(H_{1}\right) \leq$ $a(\alpha), b(\alpha) \leq a(\beta)$, and $b(\beta) \leq a(\gamma), \gamma^{*}$ is defined by $b(\gamma) \leq a\left(\gamma^{*}\right)$.

Under these conditions, a periodic solution of (1.7) of period T exists. In particular, the relation $r L\left(\gamma^{*}\right)<H_{1}-H$ is always satisfied if $r$ is sufficiently small (see Yoshizawa [3]). 


\section{Main Result}

The main result is the following theorem:

let $\tau=\max _{1 \leq i \leq n} \tau_{i}$.

Theorem 2.1. Suppose that positive constants $a, b_{i}, c, m, \delta, L_{i}$, and $\tau$ exist such that the following conditions hold:

$$
\begin{gathered}
a b-c>0, \quad f(0)=0, \quad f(x) \operatorname{sgn} x>0 \quad \forall x \neq 0, \quad \sup \left\{f^{\prime}(x)\right\}=c, \\
f(x) \operatorname{sgn} x \longrightarrow \infty \quad \text { as }|x| \longrightarrow \infty, \quad g_{i}(0)=0, \quad \frac{g_{i}(y)}{y} \geq b_{i}, \quad(y \neq 0), \\
\left|g_{i}^{\prime}(y)\right| \leq L_{i}, \quad 0 \leq \psi(y)-a \leq \delta \quad \forall y, \quad|p(\cdot)| \leq m .
\end{gathered}
$$

If

$$
\tau<\min \left\{\frac{a b-c}{(2+\mu) b N_{1}}, \frac{a b-c}{4 M_{1}}\right\}
$$

then (1.5) has a periodic solution of period $T$, where $\mu=(a b+c) / 2 b, b=\sum_{i=1}^{n} b_{i}, M_{1}=\sum_{i=1}^{n}\left(\mu L_{i} / 2\right)$ and $N_{1}=\sum_{i=1}^{n} L_{i}$.

Proof. Define a Lyapunov functional $V=V\left(x_{t}, y_{t}, z_{t}\right)$ by:

$$
V\left(x_{t}, y_{t}, z_{t}\right)=V_{1}\left(x_{t}, y_{t}, z_{t}\right)+V_{2}(x, y, z)+1+\sum_{i=1}^{n} L_{i} \int_{-\tau_{i}}^{0} \int_{t+s}^{t}|z(\theta)| d \theta d s
$$

where

$$
\begin{aligned}
V_{1}\left(x_{t}, y_{t}, z_{t}\right)= & \mu \int_{0}^{x} f(\xi) d \xi+f(x) y+\mu \int_{0}^{y} \psi(\eta) \eta d \eta+\sum_{i=1}^{n} \int_{0}^{y} g_{i}(\eta) d \eta \\
& +\mu y z+\frac{1}{2} z^{2}+\sum_{i=1}^{n} \gamma_{i} \int_{-\tau_{i}}^{0} \int_{t+s}^{t} z^{2}(\theta) d \theta d s, \\
V_{2}(x, y, z)= & \begin{cases}\frac{z}{M} \operatorname{sgn} x, & |x| \geq 1,|z| \leq M \\
\operatorname{sgn} z \operatorname{sgn} x, & |x| \geq 1,|z| \geq M \\
\frac{x z}{M}, & |x| \leq 1,|z| \leq M \\
x \operatorname{sgn} z, & |x| \leq 1,|z| \geq M\end{cases}
\end{aligned}
$$

$M,(M>1)$, and $\gamma_{i}$ are certain positive constants; the constants $\gamma_{i}$ will be determined later in the proof. 
It follows that $V_{1}(0,0,0)=0$. In view of the assumptions $\psi(y) \geq a, g_{i}(y) / y \geq b_{i}$, $(y \neq 0), f(0)=0, f(x) \operatorname{sgn} x>0,(x \neq 0)$, and $\sup \left\{f^{\prime}(x)\right\}=c$, obtain

$$
\begin{aligned}
V_{1}\left(x_{t}, y_{t}, z_{t}\right) \geq & \mu \int_{0}^{x} f(\xi) d \xi+f(x) y+\frac{1}{2} \mu a y^{2}+\sum_{i=1}^{n} \int_{0}^{y} \frac{g_{i}(\eta)}{\eta} \eta d \eta \\
& +\mu y z+\frac{1}{2} z^{2}+\sum_{i=1}^{n} \gamma_{i} \int_{-\tau_{i}}^{0} \int_{t+s}^{t} z^{2}(\theta) d \theta d s \\
\geq & \frac{1}{2 b}[b y+f(x)]^{2}+\frac{1}{2 b y^{2}}\left[4 \int_{0}^{x} f(\xi)\left\{\int_{0}^{y}\left(\mu b-f^{\prime}(\xi)\right) \eta d \eta\right\} d \xi\right] \\
& +\frac{1}{2}(\mu y+z)^{2}+\frac{1}{2} \mu(a-\mu) y^{2}+\sum_{i=1}^{n} \gamma_{i} \int_{-\tau_{i}}^{0} \int_{t+s}^{t} z^{2}(\theta) d \theta d s .
\end{aligned}
$$

Using the assumptions of Theorem 2.1, have

$$
a-\mu=a-\frac{a b+c}{2 b}>0, \quad \mu b-f^{\prime}(x) \geq \frac{a b-c}{2}>0
$$

so that

$$
V_{1}\left(x_{t}, y_{t}, z_{t}\right) \geq D_{1} x^{2}+D_{2} y^{2}+D_{3} z^{2} \geq D_{4}\left(x^{2}+y^{2}+z^{2}\right)
$$

where $D_{4}=\min \left\{D_{1}, D_{2}, D_{3}\right\}$.

It is also clear that the function $V_{2}$ is continuous and satisfies

$$
\left|V_{2}\right| \leq 1
$$

In view of (2.3), (2.7), (2.8), and the assumptions of Theorem 2.1, it can be shown that $V$ satisfies the condition (i) of Theorem 1.1.

Using a basic calculation, the time derivative of $V_{1}$ along solutions of (1.6) results in

$$
\begin{aligned}
\dot{V}_{1}\left(x_{t}, y_{t}, z_{t}\right)= & f^{\prime}(x) y^{2}+\mu z^{2}-\mu y \sum_{i=1}^{n} g_{i}(y)-\psi(y) z^{2} \\
& +(\mu y+z) \sum_{i=1}^{n} \int_{t-\tau_{i}}^{t} g_{i}^{\prime}(y(s)) z(s) d s+\sum_{i=1}^{n}\left(\gamma_{i} \tau_{i}\right) z^{2} \\
& -\sum_{i=1}^{n} \gamma_{i} \int_{t-\tau_{i}}^{t} z^{2}(s) d s+(\mu y+z) p(\cdot)
\end{aligned}
$$


The assumption $\left|g^{\prime}(y)\right| \leq L_{i}$ and the estimate $2|m||n| \leq m^{2}+n^{2}$ imply

$$
\begin{aligned}
\mu y \sum_{i=1}^{n} \int_{t-\tau_{i}}^{t} g_{i}^{\prime}(y(s)) z(s) d s & \leq \mu|y| \sum_{i=1}^{n} \int_{t-\tau_{i}}^{t}\left|g_{i}^{\prime}(y(s))\right||z(s)| d s \\
& \leq \mu|y| \sum_{i=1}^{n} \int_{t-\tau_{i}}^{t} L_{i}|z(s)| d s \\
& \leq \frac{\mu}{2} \sum_{i=1}^{n}\left(L_{i} \tau_{i}\right) y^{2}+\frac{\mu}{2} \sum_{i=1}^{n} L_{i} \int_{t-\tau_{i}}^{t} z^{2}(s) d s \\
z \sum_{i=1}^{n} \int_{t-\tau_{i}}^{t} g_{i}^{\prime}(y(s)) z(s) d s & \leq|z| \sum_{i=1}^{n} \int_{t-\tau_{i}}^{t}\left|g_{i}^{\prime}(y(s))\right||z(s)| d s \\
& \leq|z| \sum_{i=1}^{n} \int_{t-\tau_{i}}^{t} L_{i}|z(s)| d s \\
& \leq \frac{1}{2} \sum_{i=1}^{n}\left(L_{i} \tau_{i}\right) z^{2}+\frac{1}{2} \sum_{i=1}^{n} L_{i} \int_{t-\tau_{i}}^{t} z^{2}(s) d s
\end{aligned}
$$

so that

$$
\begin{aligned}
\dot{V}_{1}\left(x_{t}, y_{t}, z_{t}\right) \leq & f^{\prime}(x) y^{2}+\mu z^{2}-\mu y \sum_{i=1}^{n} g_{i}(y)-\psi(y) z^{2} \\
& +\sum_{i=1}^{n}\left(\gamma_{i} \tau_{i}\right) z^{2}+\frac{\mu}{2} \sum_{i=1}^{n}\left(L_{i} \tau_{i}\right) y^{2}+\frac{1}{2} \sum_{i=1}^{n}\left(L_{i} \tau_{i}\right) z^{2} \\
& +\sum_{i=1}^{n}\left(\frac{1}{2} L_{i}+\frac{1}{2} \mu L_{i}-\gamma_{i}\right) \int_{t-\tau_{i}}^{t} z^{2}(s) d s+(\mu y+z) p(\cdot) .
\end{aligned}
$$

Using the assumptions $\sup \left\{f^{\prime}(x)\right\}=c>0, \psi(y) \geq a$ and $a b-c>0$, and the estimation $\mu=(a b+c) / 2 b$, it follows that

$$
\begin{aligned}
\dot{V}_{1}\left(x_{t}, y_{t}, z_{t}\right) \leq & -\left[\mu \sum_{i=1}^{n} \frac{g_{i}(y)}{y}-c-\frac{\mu}{2} \sum_{i=1}^{n}\left(L_{i} \tau_{i}\right)\right] y^{2}-\left[a-\mu-\frac{1}{2} \sum_{i=1}^{n}\left(L_{i} \tau_{i}\right)-\sum_{i=1}^{n}\left(\gamma_{i} \tau_{i}\right)\right] z^{2} \\
& +\sum_{i=1}^{n}\left(\frac{1}{2} L_{i}+\frac{1}{2} \mu L_{i}-\gamma_{i}\right) \int_{t-\tau_{i}}^{t} z^{2}(s) d s|(\mu y+z)||p(\cdot)| \\
\leq & -\left[\mu \sum_{i=1}^{n} \frac{g_{i}(y)}{y}-c-\frac{\mu}{2} \sum_{i=1}^{n}\left(L_{i} \tau_{i}\right)\right] y^{2}-\left[\frac{a b-c}{2 b}-\frac{1}{2} \sum_{i=1}^{n}\left(L_{i}+2 \gamma_{i}\right) \tau_{i}\right] z^{2} \\
& +\frac{1}{2} \sum_{i=1}^{n}\left[(1+\mu) L_{i}-2 \gamma_{i}\right] \int_{t-\tau_{i}}^{t} z^{2}(s) d s+|(\mu y+z)||p(\cdot)| .
\end{aligned}
$$


If we choose $\gamma_{i}=(1 / 2) \sum_{i=1}^{n}(1+\mu) L_{i}$ and use the assumption $|p(\cdot)| \leq m$, then

$$
\begin{aligned}
\dot{V}_{1}\left(x_{t}, y_{t}, z_{t}\right) \leq & -\left[\mu \sum_{i=1}^{n} \frac{g_{i}(y)}{y}-c-\frac{\mu}{2} \sum_{i=1}^{n}\left(L_{i} \tau_{i}\right)\right] y^{2} \\
& -\left[\frac{a b-c}{2 b}-\frac{1}{2} \sum_{i=1}^{n}(2+\mu) L_{i} \tau_{i}\right] z^{2} \\
& +\mu m|y|+m|z| .
\end{aligned}
$$

An easy calculation from $V_{2}(x, y, z)$ and (1.6) leads to

$\dot{V}_{2}(\cdot)= \begin{cases}\frac{1}{M}\left\{-\psi(y) z-\sum_{i=1}^{n} g_{i}(y)-f(x)+\sum_{i=1}^{n} \int_{t-\tau_{i}}^{t} g_{i}^{\prime}(y(s)) z(s) d s+p(\cdot)\right\} \operatorname{sgn} x, & |x| \geq 1,|z| \leq M \\ 0, & |x| \geq 1,|z| \geq M \\ \frac{y z}{M}+\frac{x}{M}\left\{-\psi(y) z-\sum_{i=1}^{n} g_{i}(y)-f(x)+\sum_{i=1}^{n} \int_{t-\tau_{i}}^{t} g_{i}^{\prime}(y(s)) z(s) d s+p(\cdot)\right\}, & |x| \leq 1,|z| \leq M \\ y \operatorname{sgn} z, & |x| \leq 1,|z| \geq M\end{cases}$

so that

$$
\dot{V}_{2}(\cdot) \leq \begin{cases}-\frac{1}{M} f(x) \operatorname{sgn} x+\left\{a+\delta+m+\sum_{i=1}^{n}\left|g_{i}(y)\right|+\sum_{i=1}^{n} L_{i} \int_{t-\tau_{i}}^{t}|z(s)| d s\right\}, & |x| \geq 1,|z| \leq M \\ 0, & |x| \geq 1,|z| \geq M \\ |y|+a+\delta+m+\sum_{i=1}^{n}\left|g_{i}(y)\right|+\sum_{i=1}^{n} L_{i} \int_{t-\tau_{i}}^{t}|z(s)| d s, & |x| \leq 1,|z| \leq M \\ |y|, & |x| \leq 1,|z| \geq M,\end{cases}
$$

using the assumptions of Theorem 2.1.

First, we consider $V$ in the domain $\max \{|y|-K,|z|-M\} \geq 0$, where the constants $K$ and $M$ are large enough, which will be determined later. We have to discuss the following two cases.

Case $1^{0}(|y| \geq K \geq 1$, and $x, z$ are arbitrary). In this case, it follows that:

$$
\dot{V}_{2}(\cdot) \leq(a+\delta+m)+|y|+\sum_{i=1}^{n}\left|g_{i}(y)\right|+\sum_{i=1}^{n} L_{i} \int_{t-\tau_{i}}^{t}|z(s)| d s
$$


By the estimates (2.3), (2.13), (2.16), and $\tau=\max _{1 \leq i \leq n} \tau_{i}$, we get

$$
\begin{aligned}
\dot{V}\left(x_{t}, y_{t}, z_{t}\right) \leq & -\left[\mu \sum_{i=1}^{n} \frac{g_{i}(y)}{y}-c-\frac{\mu}{2} \sum_{i=1}^{n}\left(L_{i} \tau_{i}\right)\right] y^{2}+\sum_{i=1}^{n}\left|g_{i}(y)\right| \\
& -\left[\frac{a b-c}{2 b}-\frac{(2+\mu)}{2} \sum_{i=1}^{n}\left(L_{i} \tau_{i}\right)\right] z^{2}+(\mu m+1)|y|+m|z| \\
& +(a+\delta+m)+\sum_{i=1}^{n} L_{i} \int_{t-\tau_{i}}^{t}|z(s)| d s \\
& +\sum_{i=1}^{n}\left(L_{i} \tau_{i}\right)|z|-\sum_{i=1}^{n} L_{i} \int_{t-\tau_{i}}^{t}|z(s)| d s \\
\leq & -\left[\mu \sum_{i=1}^{n} \frac{g_{i}(y)}{y}-c-\frac{\mu}{2} \sum_{i=1}^{n}\left(L_{i} \tau_{i}\right)\right] y^{2}+\sum_{i=1}^{n}\left|g_{i}(y)\right| \\
& -\left[\frac{a b-c}{2 b}-\frac{(2+\mu)}{2} \sum_{i=1}^{n}\left(L_{i} \tau_{i}\right)\right] z^{2}+(\mu m+1)|y|+m|z| \\
& +(a+\delta+m)|y|+\sum_{i=1}^{n}\left(L_{i} \tau_{i}\right)|z| .
\end{aligned}
$$

We now consider the term

$$
\mu \sum_{i=1}^{n} g_{i}(y) y-\sum_{i=1}^{n}\left|g_{i}(y)\right|-c y^{2}-\frac{\mu}{2} \sum_{i=1}^{n}\left(L_{i} \tau_{i}\right) y^{2}
$$

and define

$$
h=\frac{a b+3 c}{2(a b+c)}<1
$$

Then, there exists a constant $K_{1},\left(K_{1}>1\right)$, satisfying $(1-1 / \mu|y|) \geq h$ for $|y| \geq K_{1}$, so that, when $|y| \geq K_{1}$,

$$
\begin{gathered}
\mu \sum_{i=1}^{n} g_{i}(y) y-\sum_{i=1}^{n}\left|g_{i}(y)\right|-c y^{2}-\frac{\mu}{2} \sum_{i=1}^{n}\left(L_{i} \tau_{i}\right) y^{2} \\
=\mu \sum_{i=1}^{n} \frac{g_{i}(y)}{y}\left(1-\frac{1}{\mu|y|}\right) y^{2}-\left(c-\sum_{i=1}^{n} \frac{\mu L_{i} \tau_{i}}{2}\right) y^{2} \\
\geq \frac{(a b+c) b}{2 b} \frac{a b+3 c}{2(a b+c)} y^{2}-\left(c-\sum_{i=1}^{n} \frac{\mu L_{i} \tau_{i}}{2}\right) y^{2}
\end{gathered}
$$


International Journal of Differential Equations

$$
\begin{aligned}
& =-\left(\frac{a b-c}{4}-\sum_{i=1}^{n} \frac{\mu L_{i} \tau_{i}}{2}\right) y^{2} \\
& \leq-\left(\frac{a b-c}{4}-M_{1} \tau\right) y^{2} .
\end{aligned}
$$

Hence

$$
\begin{aligned}
\dot{V}\left(x_{t}, y_{t}, z_{t}\right) \leq & -\left(\frac{a b-c}{4}-M_{1} \tau\right) y^{2}-\left(\frac{a b-c}{2 b}-\frac{(2+\mu) N_{1}}{2} \tau\right) z^{2} \\
& +(\mu m+1+a+\delta+m)|y|+\left(m+N_{1} \tau\right)|z|
\end{aligned}
$$

where

$$
M_{1}=\sum_{i=1}^{n} \frac{\mu L_{i}}{2}, \quad N_{1}=\sum_{i=1}^{n} L_{i}
$$

If

$$
\tau<\min \left\{\frac{a b-c}{(2+\mu) b N_{1}}, \frac{a b-c}{4 M_{1}}\right\}
$$

then the above estimate implies

$$
\dot{V}\left(x_{t}, y_{t}, z_{t}\right) \leq-\delta_{1}\left(y^{2}+z^{2}\right)+(\mu m+a+\delta+m+1)|y|+\left(m+N_{1} \tau\right)|z|
$$

for a positive constant $\delta_{1}$.

Let

$$
\rho_{1}=\max \left\{\mu m+\delta+a+m+1, m+N_{1} \tau\right\}
$$

so that

$$
\begin{aligned}
\dot{V}\left(x_{t}, y_{t}, z_{t}\right) & \leq-\delta_{1}\left(y^{2}+z^{2}\right)+\rho_{1}(|y|+|z|) \\
& =-\frac{\delta_{1}}{2}\left(y^{2}+z^{2}\right)-\frac{\delta_{1}}{2}\left[\left(|y|-\frac{\rho_{1}}{\delta_{1}}\right)^{2}+\left(|z|-\frac{\rho_{1}}{\delta_{1}}\right)^{2}-2 \frac{\rho_{1}^{2}}{\delta_{1}^{2}}\right] \\
& \leq-\frac{\delta_{1}}{2}\left(y^{2}+z^{2}\right)
\end{aligned}
$$

provided that $|y| \geq(\sqrt{2}+1) \rho_{1} \delta_{1}^{-1}$. 
Let $K=\max \left\{(\sqrt{2}+1) \rho_{1} \delta_{1}^{-1}, K_{1}\right\}$. If $|y| \geq K$, then

$$
\dot{V}\left(x_{t}, y_{t}, z_{t}\right) \leq-\frac{\delta_{1}}{2}\left(y^{2}+z^{2}\right)
$$

Case $2^{0}(|z| \geq M$, and $x, y$ are arbitrary). Then

$$
\dot{V}_{2}(x, y, z) \leq|y|
$$

By following a similar method shown in the first case, select $\gamma_{i}=(1 / 2) \sum_{i=1}^{n}(1+\mu) L_{i}$ and take

$$
\tau<\min \left\{\frac{a b-c}{(2+\mu) b N_{1}}, \frac{a b-c}{4 M_{1}}\right\}
$$

one can easily obtain

$$
\begin{aligned}
\dot{V}\left(x_{t}, y_{t}, z_{t}\right) & \leq-\delta_{2}\left(y^{2}+z^{2}\right)+(\mu m+1)|y|+\left(m+N_{1} \tau\right)|z| \\
& \leq-\delta_{2}\left(y^{2}+z^{2}\right)+\rho_{2}(|y|+|z|) \\
& \leq-\frac{\delta_{2}}{2}\left(y^{2}+z^{2}\right)
\end{aligned}
$$

for some positive constants $\delta_{2}$ and $\rho_{2}$ provided that $|z| \geq M=K$.

At the end, we consider $V$ in $\max \{|y|-K,|z|-M\} \leq 0$.

Let $|x| \geq H>1$, where the constant $H$ will be determined later. Hence

$$
\dot{V}_{2}(\cdot) \leq-\frac{1}{M} f(x) \operatorname{sgn} x+\max _{|y| \leq K} \sum_{i=1}^{n}\left|g_{i}(y)\right|+\left\{a+\delta+m+\sum_{i=1}^{n} L_{i} \int_{t-\tau_{i}}^{t}|z(s)| d s\right\} .
$$

It follows from (2.3), (2.13), and (2.31) that

$$
\begin{aligned}
\dot{V}\left(x_{t}, y_{t}, z_{t}\right) \leq & -\left(\frac{a b-c}{4}-M_{1} \tau\right) y^{2}-\left(\frac{a b-c}{2 b}-\frac{(2+\mu) N_{1}}{2} \tau\right) z^{2} \\
& +\mu m|y|+\left(m+N_{1} \tau\right)|z|-\frac{1}{M} f(x) \operatorname{sgn} x+\max _{|y| \leq K} \sum_{i=1}^{n}\left|g_{i}(y)\right| \\
& +a+\delta+m+\sum_{i=1}^{n} L_{i} \int_{t-\tau_{i}}^{t}|z(s)| d s-\sum_{i=1}^{n} L_{i} \int_{t-\tau_{i}}^{t}|z(s)| d s
\end{aligned}
$$


International Journal of Differential Equations

$$
\begin{aligned}
\leq & -\left(\frac{a b-c}{4}-M_{1} \tau\right) y^{2}-\left(\frac{a b-c}{2 b}-\frac{(2+\mu) N_{1}}{2} \tau\right) z^{2} \\
& -\frac{1}{M} f(x) \operatorname{sgn} x+\max _{|y| \leq K} \sum_{i=1}^{n}\left|g_{i}(y)\right| \\
& +\left(a+\delta+m+N_{1} M \tau+\mu m K+m M\right) .
\end{aligned}
$$

Since $f(x) \operatorname{sgn} x \rightarrow \infty$ as $|x| \rightarrow \infty$ and $|x| \geq H>1$, then we have

$$
f(x) \operatorname{sgn} x \geq 2 M\left\{\max _{|y| \leq K} \sum_{i=1}^{n}\left|g_{i}(y)\right|+a+\delta+m+N_{1} M \tau+\mu m K+m M\right\},
$$

so that

$$
-\frac{f(x) \operatorname{sgn} x}{2 M}+\left\{\max _{|y| \leq K} \sum_{i=1}^{n}\left|g_{i}(y)\right|+a+\delta+m+N_{1} M \tau+\mu m K+m M\right\} \leq 0 .
$$

In view of the above discussion, it follows that:

$$
\begin{aligned}
\dot{V}\left(x_{t}, y_{t}, z_{t}\right) \leq & -\left(\frac{a b-c}{4}-M_{1} \tau\right) y^{2}-\left(\frac{a b-c}{2 b}-\frac{(2+\mu) N_{1}}{2} \tau\right) z^{2} \\
& -\frac{1}{2 M} f(x) \operatorname{sgn} x .
\end{aligned}
$$

Subject to the evidence thus far, we can conclude that there exists a positive constant $R$, which is large enough, such that

$$
\dot{V}\left(x_{t}, y_{t}, z_{t}\right) \leq-w(u) \text { for } u^{2} \geq R^{2},
$$

where $u=\left(x^{2}+y^{2}+z^{2}\right)^{1 / 2}$. Thus, the Lyapunov functional $V\left(x_{t}, y_{t}, z_{t}\right)$ satisfies all the assumptions of Theorem 1.1. The proof for Theorem 2.1 is complete.

Example 2.2. Consider the following nonlinear differential equation of the third order with two constant deviating arguments, $\tau_{1}>0, \tau_{2}>0$ :

$$
\begin{aligned}
x^{\prime \prime \prime}+ & \left(4+\frac{1}{1+\left(x^{\prime}\right)^{2}}\right) x^{\prime \prime}+4 x^{\prime}\left(t-\tau_{1}\right)+\sin x^{\prime}\left(t-\tau_{1}\right)+4 x^{\prime}\left(t-\tau_{2}\right)+\sin x^{\prime}\left(t-\tau_{2}\right)+11 x \\
& =\frac{\sin t+\cos t}{3+\sin t+x^{2}+\cdots+x^{\prime 2}\left(t-\tau_{2}\right)+x^{\prime \prime 2}},
\end{aligned}
$$

which is a special case of (1.5). 
This equation can be written in the system form as follows:

$$
\begin{gathered}
x^{\prime}=y, \quad y^{\prime}=z, \\
z^{\prime}=-\left(4+\frac{1}{1+y^{2}}\right) z-(8 y+2 \sin y)-11 x \\
+\int_{t-\tau_{1}}^{t}(4+\cos y(s)) z(s) d s+\int_{t-\tau_{2}}^{t}(4+\cos y(s)) z(s) d s \\
+\frac{\sin t+\cos t}{3+\sin t+x^{2}+\cdots+y^{2}\left(t-\tau_{2}\right)+z^{2}} .
\end{gathered}
$$

When we compare the system described to this point with (1.6), it follows the existence of the following estimates:

$$
\begin{gathered}
\psi(y)=4+\frac{1}{1+y^{2}}, \quad 0 \leq \psi(y)-4 \leq 1, \\
a=4, \quad \delta=1, \quad g_{1}(y)=4 y+\sin y, \quad g_{1}(0)=0, \\
\frac{g_{1}(y)}{y}=4+\frac{\sin y}{y} \geq 3, \quad b_{1}=3, \\
g_{1}^{\prime}(y)=4+\cos y, \\
\left|g_{1}^{\prime}(y)\right|=|4+\cos y| \leq 5=L_{1}, \\
g_{2}(y)=4 y+\sin y, \quad g_{2}(0)=0, \\
\frac{g_{2}(y)}{y}=4+\frac{\sin y}{y} \geq 3, \quad b_{2}=3, \\
g_{2}^{\prime}(y)=4+\cos y, \\
\left|g_{2}^{\prime}(y)\right|=|4+\cos y| \leq 5=L_{2}, \\
f(x)=11 x, \quad f(0)=0, \\
f(x) \operatorname{sgn} x=11 x \operatorname{sgn} x>0, \quad(x \neq 0), \\
f(x) \operatorname{sgn} x=11 x \operatorname{sgn} x \longrightarrow \infty \quad \text { as }|x| \longrightarrow \infty, \\
f^{\prime}(x)=11, \quad a b-c=13>0, \\
h\left(t, x, x\left(t-\tau_{1}\right), \ldots, y\left(t-\tau_{2}\right), z\right) \\
=\frac{a b+3 c}{2(a b+c)}=\frac{57}{70}<1, \\
\sin t+\cos t
\end{gathered}
$$




$$
\begin{aligned}
p(t+ & \left.2 \pi, x, x\left(t-\tau_{1}\right), \ldots, y\left(t-\tau_{2}\right), z\right) \\
& =\frac{\sin (t+2 \pi)+\cos (t+2 \pi)}{3+\sin (t+2 \pi)+\cdots+z^{2}}=\frac{\sin t+\cos t}{3+\sin t+\cdots+z^{2}} \\
& =p\left(t, x, x\left(t-\tau_{1}\right), \ldots, y\left(t-\tau_{2}\right), z\right), \quad T=2 \pi .
\end{aligned}
$$

In view of the above estimates, it is seen that all the assumptions of Theorem 2.1 hold. This discussion verifies that (2.37) has a periodic solution of period $T, T=2 \pi$.

\section{Acknowledgment}

The author would like to expresses his sincere thanks and best regards to the anonymous referees for their many helpful comments, corrections, and suggestions on the paper.

\section{References}

[1] T. A. Burton, Stability and Periodic Solutions of Ordinary and Functional Differential Equations, Academic Press, Orlando, Fla, USA, 1985

[2] V. Kolmanovskii and A. Myshkis, Introduction To the Theory and Applications of Functional Differential Equations, Kluwer Academic Publishers, Dordrecht, The Netherlands, 1999.

[3] T. Yoshizawa, Stability Theory by Liapunov's Second Method, The Mathematical Society of Japan, Tokyo, Japan, 1966.

[4] E. N. Chukwu, "On the boundedness and the existence of a periodic solution of some nonlinear third order delay differential equation," Atti della Accademia Nazionale dei Lincei, Classe di Scienze Fisiche, Matematiche e Naturali, vol. 64, no. 5, pp. 440-447, 1978.

[5] Y. F. Zhu, "On stability, boundedness and existence of periodic solution of a kind of third order nonlinear delay differential system," Annals of Differential Equations, vol. 8, no. 2, pp. 249-259, 1992.

[6] H. O. Tejumola and B. Tchegnani, "Stability, boundedness and existence of periodic solutions of some third and fourth order nonlinear delay differential equations," Journal of the Nigerian Mathematics Society, vol. 19, pp. 9-19, 2000.

[7] C. Tunç, "On the existence of periodic solutions to nonlinear third order ordinary differential equations with delay," Journal of Computational Analysis and Applications, vol. 12, no. 1, pp. 191-201, 2010. 


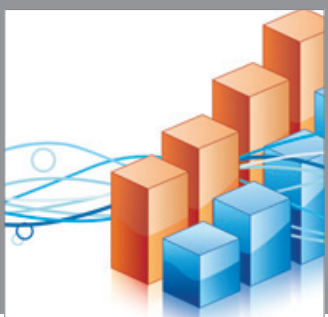

Advances in

Operations Research

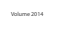

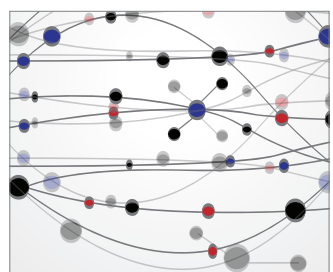

\section{The Scientific} World Journal
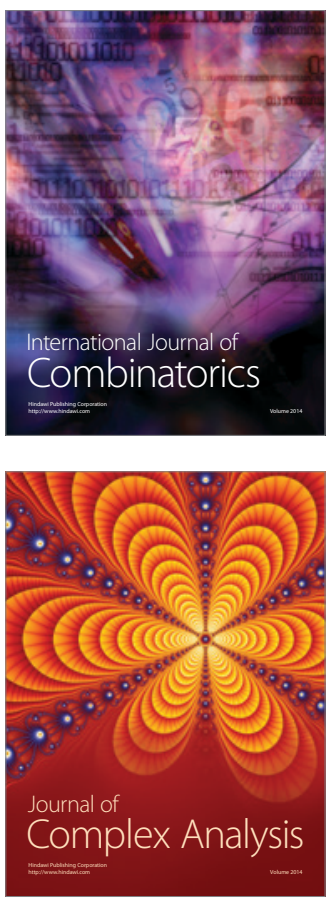

International Journal of

Mathematics and

Mathematical

Sciences
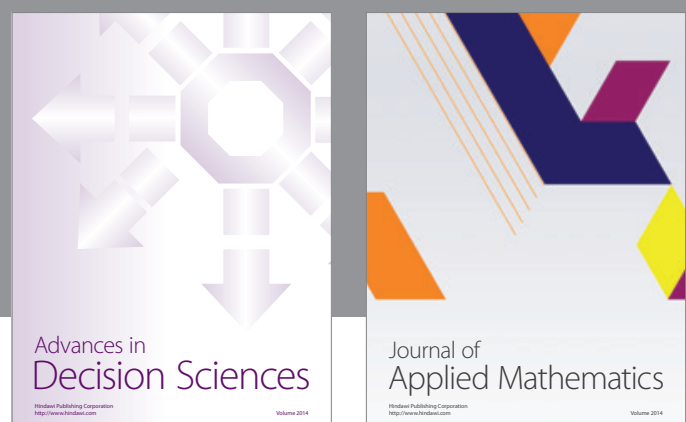

Journal of

Applied Mathematics
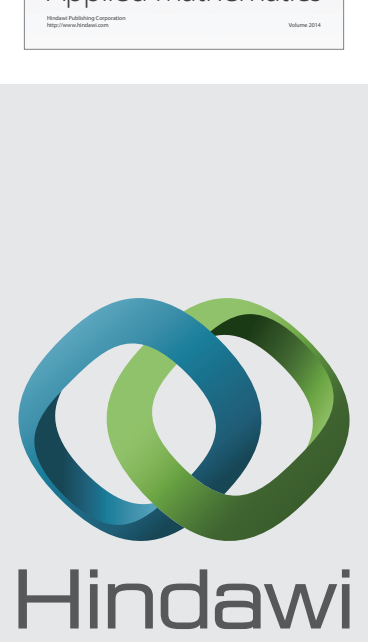

Submit your manuscripts at http://www.hindawi.com
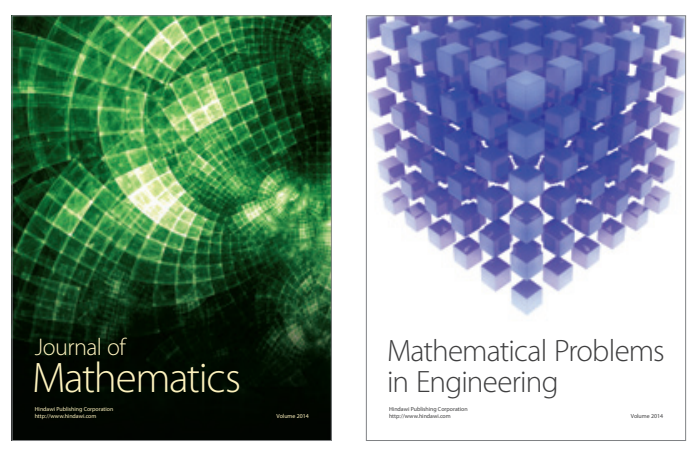

Mathematical Problems in Engineering
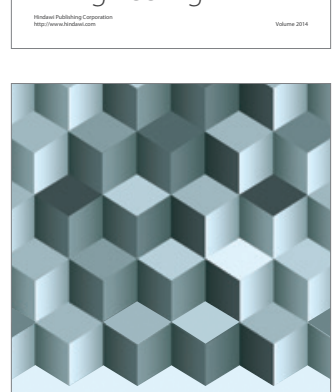

Journal of

Function Spaces
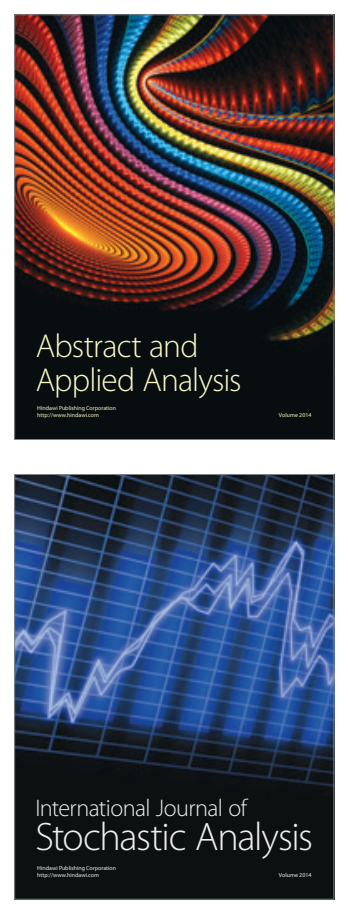

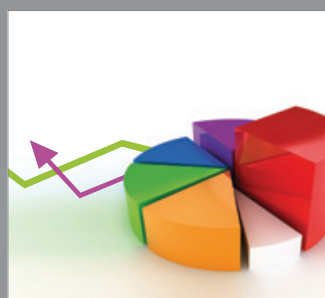

ournal of

Probability and Statistics

Promensencen
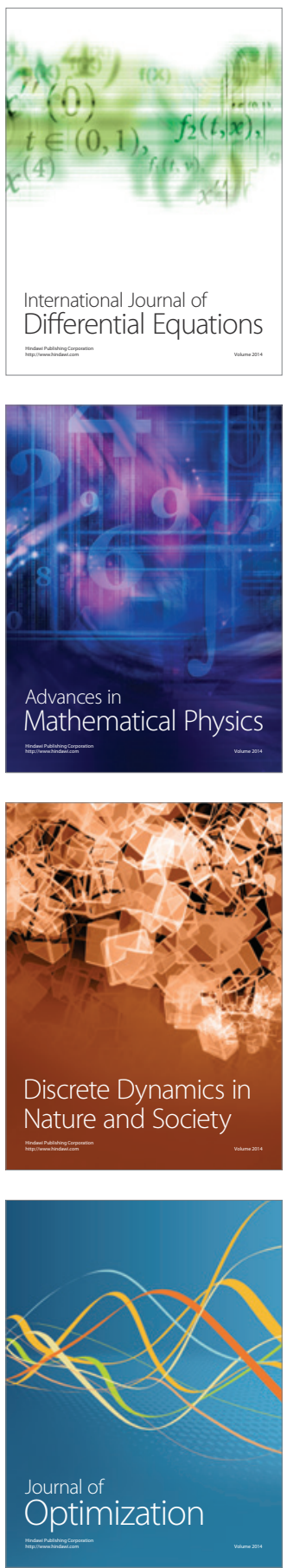